Article

\title{
Active Pore-Edge Engineering of Single-Layer Niobium Diselenide Porous Nanosheets Electrode for Hydrogen Evolution
}

\author{
Jianxing Wang, Xinyue Liu $®$, Ying Liu $®$ and Guowei Yang * \\ State Key Laboratory of Optoelectronic Materials and Technologies, Nanotechnology Research Center, \\ School of Materials Science \& Engineering, School of Physics, Sun Yat-sen University, \\ Guangzhou 510275, Guangdong, China; wangjx46@mail2.sysu.edu.cn (J.W.); liuxiny8@mail2.sysu.edu.cn (X.L.); \\ liuying35@mail.sysu.edu.cn (Y.L.) \\ * Correspondence: stsygw@mail.sysu.edu.cn; Tel.: +86-20-8411-3692
}

Received: 6 March 2019; Accepted: 1 May 2019; Published: 16 May 2019

check for updates

\begin{abstract}
Two-dimensional transition-metal dichalcogenides (TMDs) possess interesting catalytic properties for the electrochemical-assisted hydrogen-evolution reaction (HER). We used niobium diselenide $\left(\mathrm{NbSe}_{2}\right)$ as a representative TMD, and prepared single-layer $\mathrm{NbSe}_{2}$ porous nanosheets (PNS) by a double-sonication liquid-phase exfoliation, with $\mathrm{H}_{2} \mathrm{O}_{2}$ as a pore-forming agent. The single-layer $\mathrm{NbSe}_{2}$ PNS were drop-cast on carbon foam (CF) to fabricate a three-dimensional robust $\mathrm{NbSe}_{2}$ $\mathrm{PNS} / \mathrm{CF}$ electrode. The $\mathrm{NbSe}_{2}$ PNS/CF electrode exhibits a high current density of $-50 \mathrm{~mA} \mathrm{~cm}^{-2}$ with an overpotential of $148 \mathrm{mV}$ and a Tafel slope of $75.8 \mathrm{eV} \mathrm{dec}^{-1}$ for the HER process. Little deactivation is detected in continuous $\mathrm{CV}$ testing up to 20,000 cycles, which suggests that this novel $\mathrm{NbSe}_{2} \mathrm{PNS} / \mathrm{CF}$ is a promising catalytic electrode in the HER application. The porous structure of single-layer $\mathrm{NbSe}_{2}$ nanosheets can enhance the electrochemical performance compared with that of pore-free $\mathrm{NbSe}_{2}$ nanosheets. These findings illustrate that the single-layer $\mathrm{NbSe}_{2} \mathrm{PNS}$ is a potential electrocatalytic material for HER. More importantly, the electrochemical performance of the $\mathrm{NbSe}_{2} \mathrm{PNS} / \mathrm{CF}$ expands the use of two-dimensional TMDs in electrocatalysis-related fields.
\end{abstract}

Keywords: TMDs; niobium diselenide; hydrogen evolution; electrocatalysis

\section{Introduction}

The energy crisis has aroused extensive research interest in the search for sustainable energy-conversion systems that exhibit a high productivity and low cost. Hydrogen $\left(\mathrm{H}_{2}\right)$ is one of the most promising candidates to replace fossil fuels in the future [1-8]. The electrochemical hydrogen-evolution reaction (HER) is considered to be the most important and promising route to produce hydrogen [9-13]. Platinum $(\mathrm{Pt})$ and its alloys are the most electrochemically active and stable catalysts for HER. However, the high price and limited availability of Pt prevent its large-scale usage in practice [14]. Therefore, the development of nonprecious-metal catalysts that drive HER at a low overpotential with an excellent reaction efficiency is essential for large-scale production of hydrogen through electrochemical water splitting $[15,16]$.

Recently, two-dimensional (2D) transition-metal dichalcogenides (TMDs), such as $\mathrm{MoS}_{2}$ and $\mathrm{WS}_{2}$, have attracted much attention because of their layer structure and excellent electrocatalytic properties [17]. The inherent contact resistance of TMD materials has not yet been optimized, especially for the trigonal prismatic $(2 \mathrm{H})$ basal plane. The crystalline strain and metallic octahedral (1T) sites are both important factors to modulate the catalytic activity of TMD nanosheets [18-20]. Therefore, an improvement of the conductivity and creation of active edge sites of the TMDs are expected to achieve a better HER performance. 
In the thermodynamically stable $2 \mathrm{H}$ phase, $\mathrm{MoS}_{2}, \mathrm{MoSe}_{2}, \mathrm{WS}_{2}$ and $\mathrm{WSe}_{2}$ are semiconductors [21]. The $\mathrm{NbSe}_{2}$ belongs to the Group $\mathrm{V}$ transition metal dichalcogenides. $\mathrm{NbSe}_{2}$ has a similar crystalline structure to $\mathrm{MoS}_{2}$ and $\mathrm{WS}_{2}$. However, the $\mathrm{NbSe}_{2} \mathrm{TMDs}$ with metallic conductivity have stolen the limelight [22]. The electrical resistivity of $\mathrm{NbSe}_{2}$ is only $10^{-4} \Omega \cdot \mathrm{cm}$, which is six orders of magnitude less than that of $\mathrm{MoS}_{2}$ [23]. The Group V NbSe${ }_{2}$ TMDs are prized for their low-dimensional crystal structure and exhibit interesting electronic properties, such as superconductivity, charge density waves and Mott transition [24]. The layers of $\mathrm{NbSe}_{2}$ are stacked together through Van der Waals interactions and can be exfoliated into thin layers. First-principles calculations have suggested that single-layer $\mathrm{NbSe}_{2}$ has a charge density wave phase with a different periodicity compared with that of the bulk, as well as a larger gain of electronic energy, which result in a higher transition temperature [25]. However, the electrochemical and electrocatalytic properties of the single-layer Group V TMDs have not been well established.

Here, we prepared 3D single-layer $\mathrm{NbSe}_{2}$ porous nanosheets as advanced HER electrocatalysts. Strategies have been developed to promote the HER catalytic effect of $\mathrm{NbSe}_{2}$, which can increase the number of edge active sites significantly [4,26,27] and improve the electrical conductivity [28]. We fabricated single-layer $\mathrm{NbSe}_{2}$ porous nanosheets/carbon-foam electrode, which exhibited a Tafel slope of $75.8 \mathrm{eV} \mathrm{dec}^{-1}$ and an overpotential of $-148 \mathrm{mV}$ at a current density of $-50 \mathrm{~mA} \mathrm{~cm}^{-2}$ in the HER process. The as-revealed catalytic performance of a single-layer $\mathrm{NbSe}_{2} \mathrm{PNS} / \mathrm{carbon}$ foam (CF) electrode outperforms most of the previously reported non-noble HER catalysts, such as $\mathrm{MoS}_{2}-\mathrm{NbSe}_{2}$ hybrid nanobelts with a Tafel slope of $79.5 \mathrm{eV} \mathrm{dec}^{-1}$ and an overpotential of $-410 \mathrm{mV}$ at a current density of $-10 \mathrm{~mA} \mathrm{~cm}^{-2}$ [20], three-dimensional molybdenum sulfide sponges with a Tafel slope of $185 \mathrm{eV} \mathrm{dec}^{-1}$ and an overpotential of $-30 \mathrm{mV}$ at a current density of $-10 \mathrm{~mA} \mathrm{~cm}^{-2}$ [29], and a three-dimensional $\mathrm{MoS}_{2} / \mathrm{GO}$ framework with an overpotential of $-210 \mathrm{mV}$ at a current density of $-10 \mathrm{~mA} \mathrm{~cm}^{-2}$ [30]. Little deactivation has been detected in stability testing, even up to 20,000 cycles, which reveals the promising prospect of this novel single-layer $\mathrm{NbSe}_{2}$ porous nanosheets/carbon in massive electrochemical water splitting and hydrogen production.

\section{Materials and Methods}

$\mathrm{NbSe}_{2}$ pristine powder (99\%, Alfa Aesar, Shanghai, China), sodium cholate (NaC) (99\%, Alfa Aesar, Shanghai, China), $\mathrm{Nb}_{2} \mathrm{O}_{5}$ powder (99.99\%, Alfa Aesar, Shanghai, China), carbon foam, Nafion solution (5 wt.\%, Alfa Aesar, Shanghai, China), and state-of-the-art Pt-C (10 wt.\% Pt, Alfa Aesar, Shanghai, China). Other chemicals were from SinoPharm (Shanghai, China) and used without further purification.

$\mathrm{NbSe}_{2}$ powder (starting concentration $\mathrm{C}_{\mathrm{i}}=8 \mathrm{mg} \mathrm{mL}^{-1}$ ) was dissolved in $200 \mathrm{~mL}$ of aqueous $\mathrm{NaC}$ solution $\left(\mathrm{C}_{\mathrm{NaC}}=4 \mathrm{mg} \mathrm{mL}{ }^{-1}\right)$. To obtain a stable single-layer nanosheets dispersion and to avoid re-stacking, the initial mass ratio of $\mathrm{NaC}$ to $\mathrm{TMD}\left(\mathrm{C}_{\mathrm{NaC}} / \mathrm{C}_{\mathrm{i}}\right)$ was kept at $\sim 0.5$, which is the optimized surfactant concentration ratio to drive efficient exfoliation. The initial dispersion was sonicated for $6 \mathrm{~h}$ at a $30 \%$ amplitude under pulsed mode with $2 \mathrm{~s}$ on and $2 \mathrm{~s}$ off while chilled using a double-jacketed water-cooling system and chiller. The resultant raw dispersions were subjected to a brief centrifugation step (TGL-16 centrifuge, Xiangyi Co. Ltd., Hunan, China) at 5000 rpm for 50 min to remove un-exfoliated material. The upper suspension was then subjected to another centrifugation step at 10,000 rpm for $30 \mathrm{~min}$ to separate the single-layer nanosheets (NSs) from the few-layer NSs. The collected single-layer nanosheets were sonicated again using the same conditions, except in a $2.5 \mathrm{vol} \% \mathrm{H}_{2} \mathrm{O}_{2}$ to create pores on the nanosheets. Finally, the $\mathrm{NbSe}_{2}$ single-layer PNS were rinsed with $1200 \mathrm{~mL}$ of water to remove residual surfactant and $\mathrm{H}_{2} \mathrm{O}_{2}$ during the vacuum filtration.

Scanning electron microscopy (SEM, Auriga-4525, Carl Zeiss Inc., Oberkochen, Germany) and transmission electron microscopy (TEM, Tecnai G2 F30, FEI Inc., Eindhoven, The Netherlands, operated at $300 \mathrm{kV}$ ) were used to identify the morphology of the as-synthesized samples. To understand the surface chemical states of the superficial bonded elements, X-ray photoelectron spectroscopy with a monochromatic Al-K $\alpha$ source (XPS, ESCA Lab250. Thermo Scientific, East Grinstead, UK) was 
conducted. Ultraviolet photoelectron spectroscopy (UPS, VG ESCALAB Mk II, Thermo Scientific, East Grinstead, UK) was performed using He I (21.2 eV) resonance line. The X-ray diffraction (XRD) pattern was recorded on a Rigaku D-MAX 2200 VPC (Rigaku Co., Tokyo, Japan) diffractometer with Cu-K $\alpha$ as the radiation source $(\lambda=0.154 \mathrm{~nm})$. Atomic force microscopy (AFM) images were obtained by using a Bruker Multimode V8 system (Dimension icon, Bruker Inc., Billerica, MA, USA) with the tapping mode after the samples had been deposited on a freshly cleaved mica surface by spin coating.

Typically, $5 \mathrm{mg}$ of sample and $30 \mu \mathrm{L}$ of Nafion solution ( $5 \mathrm{wt}$.\%) were dispersed uniformly in $1 \mathrm{~mL}$ of a water-ethanol solution with a volume ratio of $4: 1$ by sonicating for $0.5 \mathrm{~h}$ to form a homogeneous ink. Then, $100 \mu \mathrm{L}$ catalyst ink was loaded onto a carbon-foam electrode with a geometric area of $0.5 \mathrm{~cm}^{-2}$. The catalytic performances of the single-layer $\mathrm{NbSe}_{2} \mathrm{PNS} / \mathrm{CF}$ for HER were studied using a three-electrode configuration connected to a $\mathrm{CH}$ Instrument workstation at room temperature $\left(25^{\circ} \mathrm{C}\right)$. The $\mathrm{NbSe}_{2}$ PNS/CF electrode was used as the working electrode. An Ag/AgCl (sat. $\mathrm{KCl}$ ) electrode and a graphite rod were used as the reference and counter electrodes, respectively. All measurements were performed in $0.5 \mathrm{M} \mathrm{H}_{2} \mathrm{SO}_{4}$ (aq.). All reported potentials were referenced to the reversible hydrogen electrode (RHE) through RHE calibration according to: $\mathrm{E}(\mathrm{RHE})=\mathrm{E}^{\theta}(\mathrm{Ag} / \mathrm{AgCl}$ sat. $)+0.198+0.059 \mathrm{pH}$. The polarization curves were obtained by sweeping the potential from -0.4 to $0.2 \mathrm{~V}$ versus the RHE at room temperature with a sweep rate of $5 \mathrm{mV} \mathrm{s}^{-1}$. The electrochemical impedance spectroscopy (EIS) measurements were performed in the same configuration at an open circuit potential of $210 \mathrm{mV}$ over a frequency range from $100 \mathrm{kHz}$ to $0.1 \mathrm{~Hz}$ at an amplitude of $2 \mathrm{mV}$. The resistance of $0.5 \mathrm{M} \mathrm{H}_{2} \mathrm{SO}_{4}$ is $\sim 15 \Omega$, which was determined by EIS.

\section{Results}

Liquid-phase sonication exfoliation is a powerful and scalable technique to produce few-layer TMD nanosheets [31-35]. Figure 1 illustrates the fabrication process of the single-layer $\mathrm{NbSe}_{2} \mathrm{PNS}$. Firstly, the $\mathrm{NbSe}_{2}$ pristine powders were exfoliated into few-layer nanosheets through the sonication liquid-phase exfoliation process. Secondly, porous structures in the plane of the prepared nanosheets were constructed through a second liquid-phase sonication process in $\mathrm{H}_{2} \mathrm{O}_{2}$. After double liquid-phase sonication, $\mathrm{NbSe}_{2}$ crystals in the powder were exfoliated into single-layer PNS.

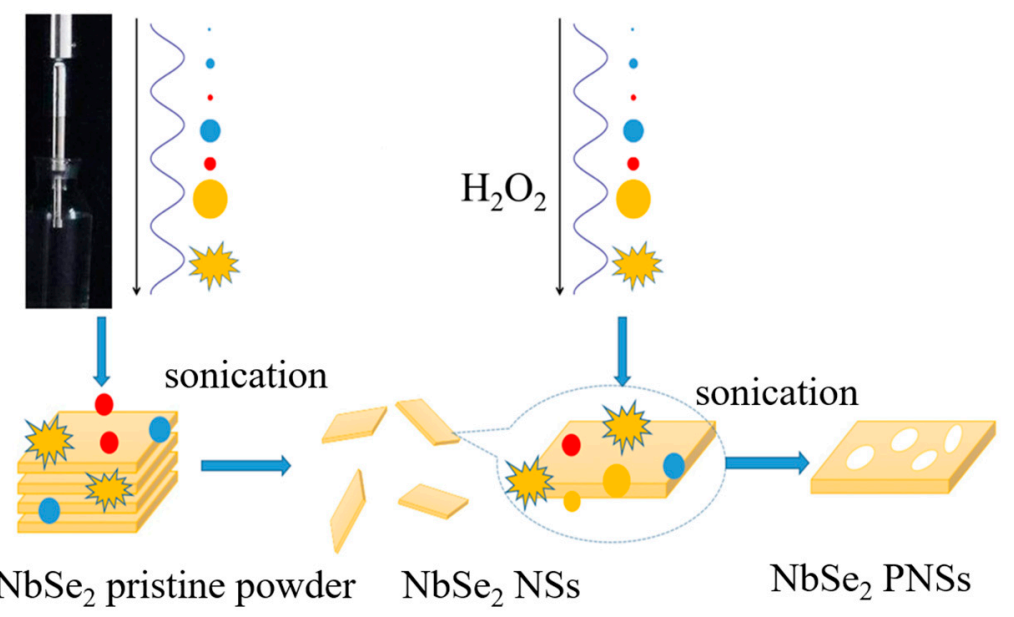

Figure 1. Schematic illustration of the process to prepare the single-layer porous $\mathrm{NbSe}_{2}$ nanosheets.

$\mathrm{NbSe}_{2}$ powders tend to have a bulk structure with fewer edge sites, but single-layer $\mathrm{NbSe}_{2} \mathrm{PNS}$ are almost $1 \mathrm{~nm}$ thickness and contain many holes in the plane. Therefore, the edge active sites of single-layer $\mathrm{NbSe}_{2} \mathrm{PNS}$ are several orders of magnitude higher than those of the $\mathrm{NbSe}_{2}$ powders. The $\mathrm{NbSe}_{2}$ PNS solution which mixed with Nafion was drop-casted on the carbon foam (CF) to fabricate the $\mathrm{NbSe}_{2} \mathrm{PNS} / \mathrm{CF}$ electrode (Figure 2). The macropore-like structure of CF could increase the contact area of catalyst and electrolyte as well as improve the electrochemical property of the $\mathrm{NbSe}_{2} \mathrm{PNS}_{\text {[36] }}$. 


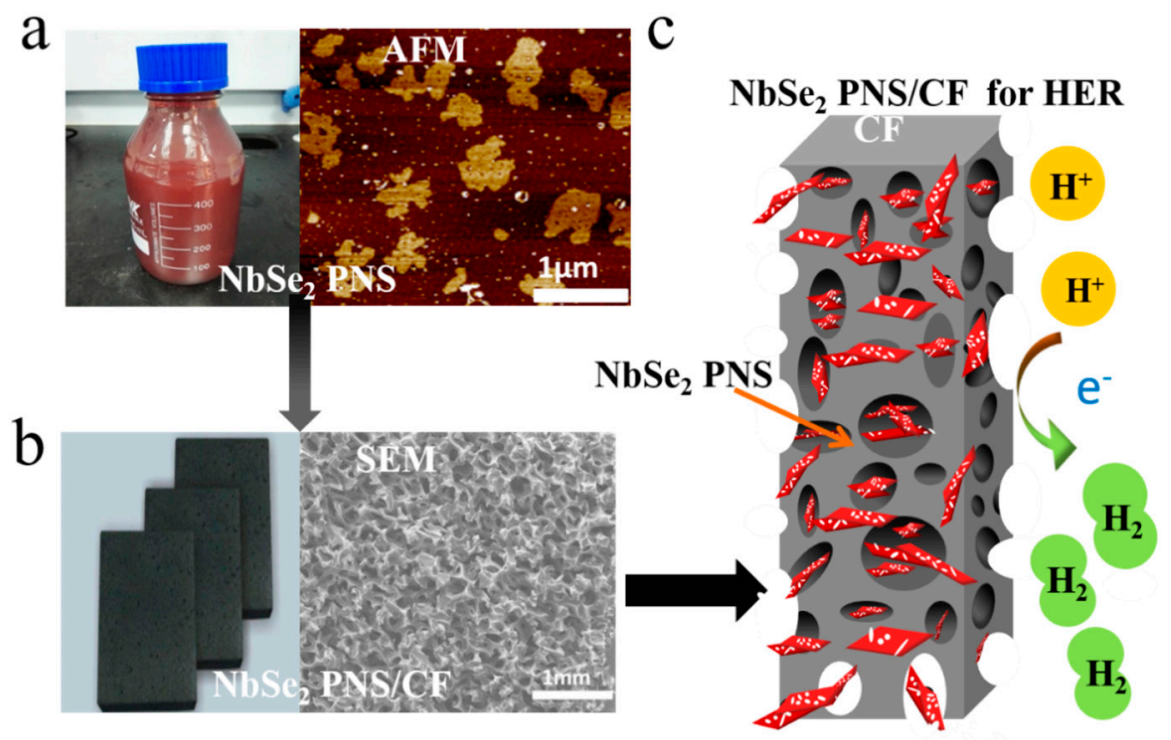

Figure 2. (a) Photograph of well dispersed $\mathrm{NbSe}_{2}$ porous nanosheets (PNS) solution and AFM image of scattered $\mathrm{NbSe}_{2}$ PNS. (b) Optical photograph and corresponding SEM image of as-developed $\mathrm{NbSe}_{2} \mathrm{PNS} /$ carbon foam (CF) electrode. (c) Illustration of the $\mathrm{NbSe}_{2} \mathrm{PNS} / \mathrm{CF}$ electrode toward the hydrogen-evolution reaction (HER).

To confirm this hypothesis, single-layer and porous nanosheets were investigated via X-ray powder diffraction (XRD) to identify the corresponding crystal structure. According to Figure 3a, several peaks of bulk $\mathrm{NbSe}_{2}$ are assigned to the hexagonal $2 \mathrm{H}-\mathrm{NbSe}_{2}$ (JCPDS 65-3484). As a comparison, the $\mathrm{NbSe}_{2}$ PNS exhibit an obvious diffraction peak at $14.1^{\circ}$, which is related to the (002) peak of hexagonal $\mathrm{NbSe}_{2}$. In addition, the (002) peak of $\mathrm{NbSe}_{2}$ PNS shifts to the higher angle compared to that of bulk $\mathrm{NbSe}_{2}$ due to the partial transformation from $2 \mathrm{H}-\mathrm{NbSe}_{2}$ to $1 \mathrm{~T}-\mathrm{NbSe}_{2}$ (Figure $3 \mathrm{~b}$ ). The similar phenomenon of $2 \mathrm{H}$ and $1 \mathrm{~T} \mathrm{MoS}_{2}$ monolayer has been reported in literature [37]. Besides this, the positive shift peak of $\mathrm{NbSe}_{2}$ PNS suggests the highly exfoliated effect of the $\mathrm{NbSe}_{2}$ nanosheets and the widened interlayer spacing of $\mathrm{NbSe}_{2}$ PNS owing to the strong exfoliating ability with the assistance of $\mathrm{H}_{2} \mathrm{O}_{2}$.
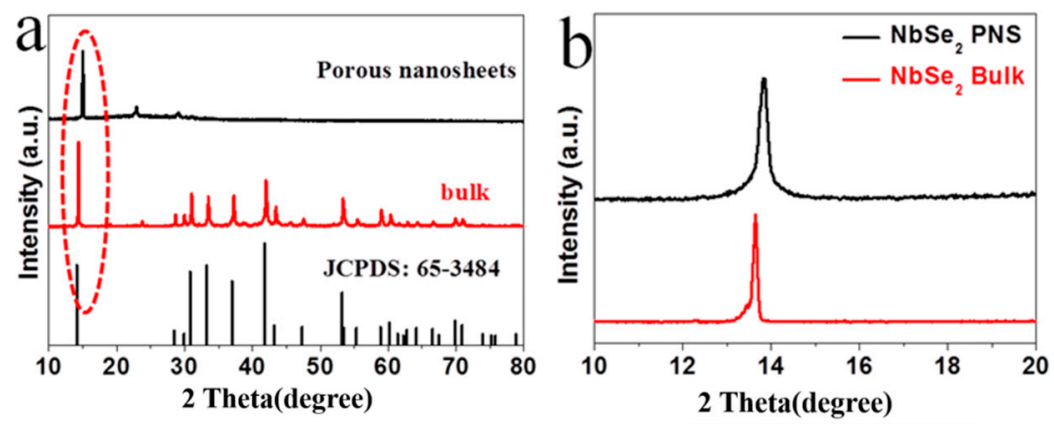

Figure 3. XRD patterns of (a) $\mathrm{NbSe}_{2} \mathrm{PNS}$ and pristine powder $\mathrm{NbSe}_{2}$; (b) the amplifying district of circle in (a).

Figure 4a and Figure S1a reveal that the CF support exhibits a network porous structure, and the size of the holes ranges from dozens to hundreds of micrometers. The initial $\mathrm{NbSe}_{2}$ pristine powder is in the form of crystalline flakes with size of a few to dozens of micrometers (Figure S1b). Furthermore, the size of the $\mathrm{NbSe}_{2}$ PNS are smaller than that of the $\mathrm{NbSe}_{2} \mathrm{NS}$ (Figure S1c,d), suggesting that the second liquid-phase sonication further broke the $\mathrm{NbSe}_{2}$ nanosheets to smaller pieces. The elemental distributions indicate the uniform distribution of elemental $\mathrm{C}, \mathrm{Nb}$ and $\mathrm{Se}$ on the $3 \mathrm{D} \mathrm{NbSe} \mathrm{NNS}_{2} \mathrm{PFF}$ surface, meaning that the $\mathrm{NbSe}_{2}$ PNS have been attached onto the CF (Figure $4 \mathrm{~d}-\mathrm{f}$ ). 


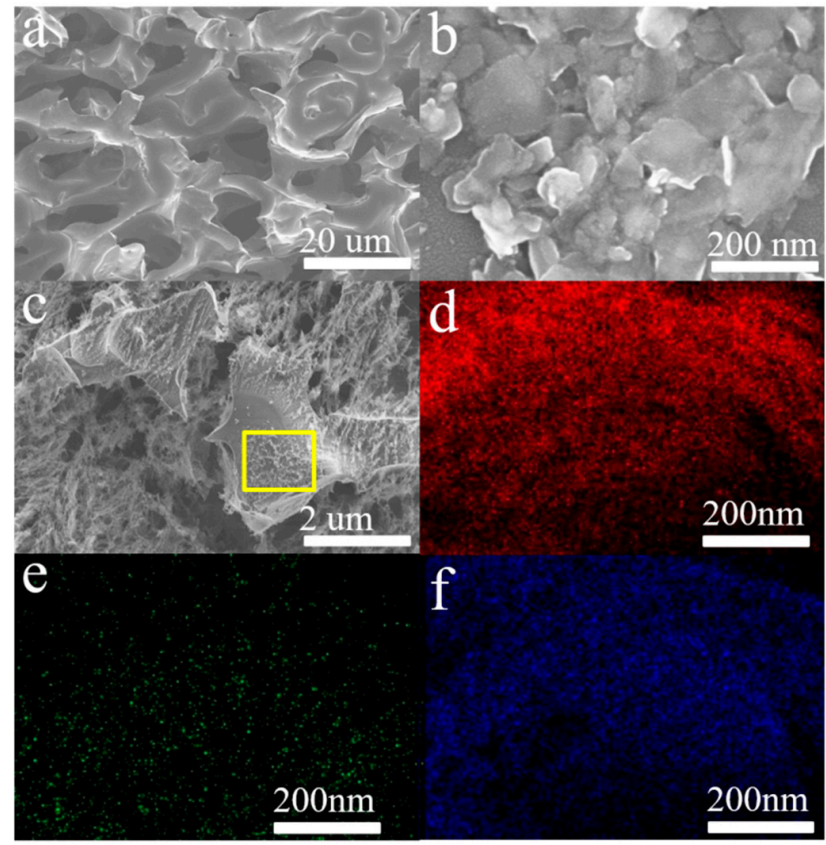

Figure 4. SEM images of the (a) bare carbon foam, (b) as-synthesized $\mathrm{NbSe}_{2} \mathrm{PNS}$ and (c) $3 \mathrm{D} \mathrm{NbSe}$ PNS/CF. (d-f) EDS mapping of $\mathrm{Nb}$, Se, and C elements on the surface of $\mathrm{NbSe}_{2} \mathrm{PNS} / \mathrm{CF}$ (the region marked in (c)).

The thickness of the $\mathrm{NbSe}_{2}$ PNS was investigated by atomic force microscopy (AFM) and TEM. The AFM results in Figure $5 \mathrm{a}, \mathrm{b}$ confirmed that single-layer $\mathrm{NbSe}_{2}$ PNS with thickness of $\sim 1 \mathrm{~nm}$ were obtained. In comparison, the $\mathrm{NbSe}_{2} \mathrm{NSs}$ thickness $(\sim 1.5 \mathrm{~nm})$ is larger than the $\mathrm{NbSe}_{2}$ PNS (Figure S2d). The porous structure can also be clearly seen from Figure $5 \mathrm{c}$, due to the etching effect of $\mathrm{H}_{2} \mathrm{O}_{2}$. The size of the nanosheets' hole ranges from several to dozens of nanometers. The high-resolution TEM (HRTEM) image (Figure 4d) shows the lattice fringe of $0.31 \mathrm{~nm}$ was resulting from the (002) crystal planes of $\mathrm{NbSe}_{2}$. However, as shown in Figure S2, $\mathrm{NbSe}_{2} \mathrm{NSs}$ without etching by $\mathrm{H}_{2} \mathrm{O}_{2}$ do not exhibit a hole structure in the nanosheets. Relative to the $\mathrm{NbSe}_{2}$ powder, the unsaturated edges of the exfoliated porous $\mathrm{NbSe}_{2}$ PNS structure are more active for proton adsorption and thus enhance the HER performance [28].

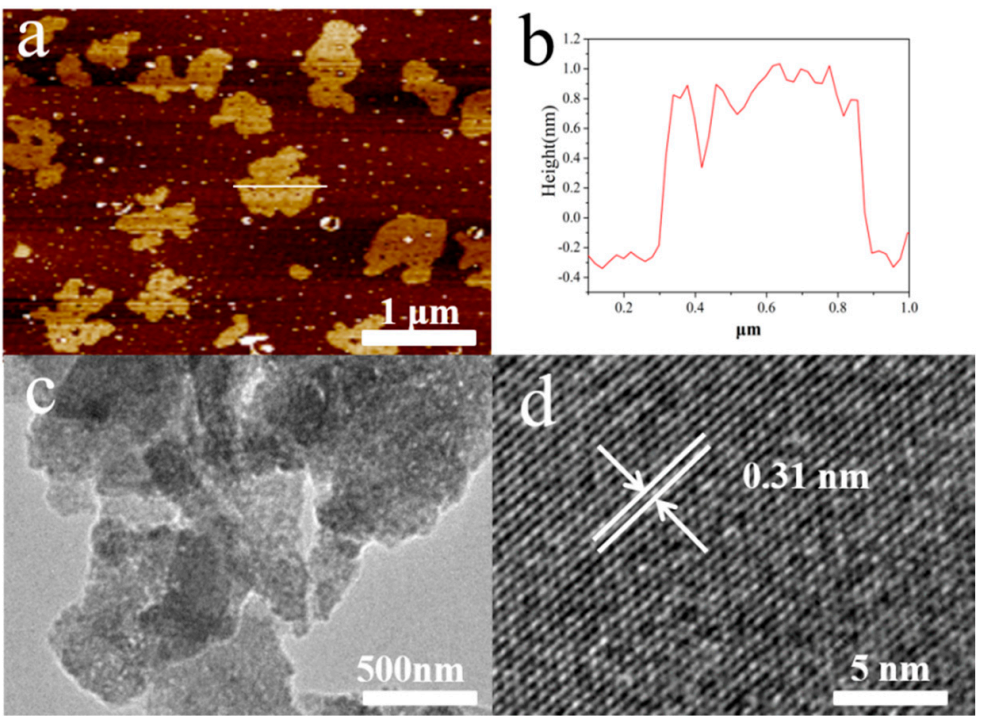

Figure 5. (a) AFM image and (b) the corresponding thickness distribution of $\mathrm{NbSe}_{2}$ PNS. (c) TEM and (d) HR-TEM images of $\mathrm{NbSe}_{2}$ PNS. 
XPS was used to characterize the chemical composition and binding energy of the single-layer $\mathrm{NbSe}_{2} \mathrm{PNS}$ and $\mathrm{NbSe}_{2} \mathrm{NSs}$. The XPS spectrum of $\mathrm{Nb} 3 \mathrm{~d}$ for the single-layer $\mathrm{NbSe}_{2} \mathrm{PNS}$ is shown in Figure 6a. The high-resolution spectrum shows 1T-NbSe 2 peaks (blue line) around 203.2 and $206.0 \mathrm{eV}$, which corresponds to the $\mathrm{Nb}^{4+} 3 \mathrm{~d}$ component. $2 \mathrm{H}-\mathrm{NbSe}_{2}$ peaks (dark yellow line) at around 204.1 and $206.5 \mathrm{eV}$ correspond to the $\mathrm{Nb}^{4+} 3 \mathrm{~d}$ component. Peaks around 207.8 and $210.1 \mathrm{eV}$ are attributed to $\mathrm{Nb}^{5+}$, indicating that oxidized valence of $\mathrm{Nb}^{5+}$ exists at the surface of nanosheets. The high-resolution XPS spectra of $\mathrm{O} 1 \mathrm{~s}$ of $\mathrm{NbSe}_{2}$ PNS (Figure S7) shows two peaks at 529.6 and $532.0 \mathrm{eV}$, which are due to the lattice oxygen and the adsorption oxygen in the surface of catalyst, respectively. The high-resolution XPS spectrum of Se $3 \mathrm{~d}$ for the single-layer $\mathrm{NbSe}_{2}$ PNS is shown in Figure $6 \mathrm{~b}$. Peaks at around $52.85 \mathrm{eV}$ and $53.65 \mathrm{eV}$ are attributed to Se $3 \mathrm{~d}_{5 / 2}$ and Se $3 \mathrm{~d}_{3 / 2}$ of $1 \mathrm{~T}-\mathrm{NbSe}_{2}$, respectively. Another two peaks at $54.9 \mathrm{eV}$ and $55.7 \mathrm{eV}$ can be assigned to $\mathrm{Se} 3 \mathrm{~d}_{5 / 2}$ and $\mathrm{Se} 3 \mathrm{~d}_{3 / 2}$ of $2 \mathrm{H}-\mathrm{NbSe}_{2}$ [38]. In comparison, according to $\mathrm{Se} 3 \mathrm{~d}$ core-level peaks of the $\mathrm{NbSe}_{2} \mathrm{NSs}$ in Figure $\mathrm{S} 3 \mathrm{a}, 2 \mathrm{H}-\mathrm{NbSe}_{2}$ peaks around $54.7 \mathrm{eV}$ and $55.7 \mathrm{eV}$ should be related to Se $3 \mathrm{~d}_{3 / 2}$ and Se $3 \mathrm{~d}_{5 / 2}$. The $\mathrm{Nb} 3 \mathrm{~d}$ core-level peaks of the $\mathrm{NbSe}_{2} \mathrm{NSs}$ at $203.5 \mathrm{eV}$ and $206.6 \mathrm{eV}$ in Figure S3b should represent the $\mathrm{Nb}^{4+} 3 \mathrm{~d}$ component. However, the XPS of $\mathrm{NbSe}_{2}$ NSs is not detected in the $1 \mathrm{~T}$ phase in the nanosheets, which shows that part of the $2 \mathrm{H}$ phase can be transformed to the $1 \mathrm{~T}$ phase during the second sonication process with the assistance of $\mathrm{H}_{2} \mathrm{O}_{2}$ [38]. The UPS of single-layer $\mathrm{NbSe}_{2}$ PNS was shown in Figure 6c. The working function $(\Phi)$ of $\mathrm{NbSe}_{2}$ PNS was calculated as $4.18 \mathrm{eV}$. When kinetic energy is used as the $x$-axis, the equation of the working function is $\Phi=h \gamma-\left(E_{\text {Fermi, }}-E_{S E}\right.$ Cutoff, $\left.k\right)$. The photon energy of XPS monochromatic is $1.486 \mathrm{eV}$ and $\mathrm{E}_{\mathrm{Fermi}, \mathrm{k}}$ is $1.486 \mathrm{eV}$. Hence, the value of $\Phi$ is equal to the value of $\mathrm{E}_{\mathrm{SE}}$ Cutoff, $\mathrm{k}$.
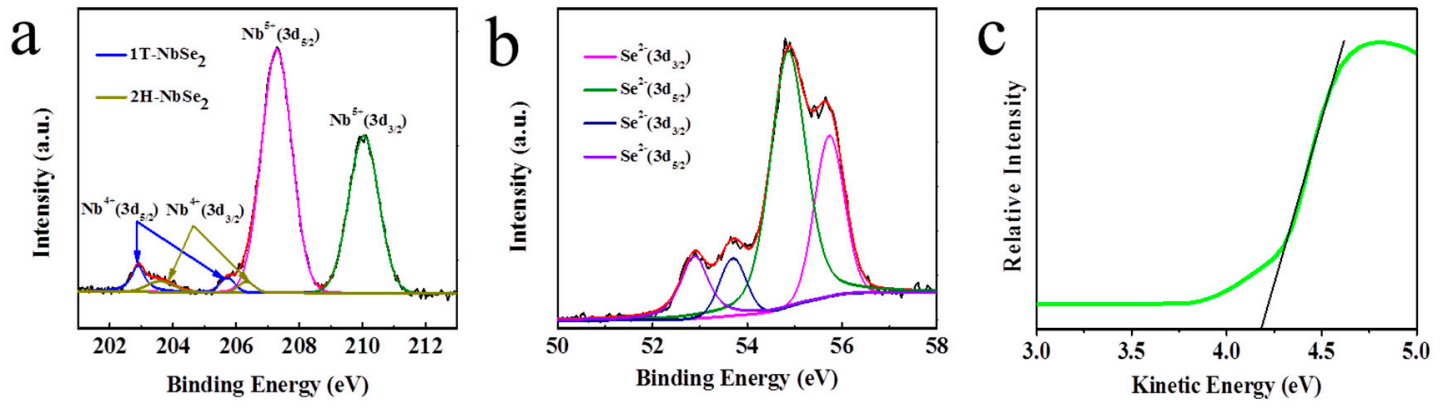

Figure 6. The high-resolution XPS spectra of single-layer $\mathrm{NbSe}_{2}$ PNS. (a) Se 3d and (b) Nb 3d spectra. (c) UPS spectrum of single-layer $\mathrm{NbSe}_{2}$ PNS.

The XPS spectra of $\mathrm{NbSe}_{2}$ PNS after 50 consecutive cyclic voltammetry sweeps and $\mathrm{NbSe}_{2} \mathrm{PNS}$ after $25 \mathrm{~h}$ stability test were shown in Figure S8. The related XPS analysis results of $\mathrm{NbSe}_{2} \mathrm{PNS}_{1} \mathrm{NbSe}_{2}$ PNS after 50 consecutive cyclic voltammetry sweeps, as well as $\mathrm{NbSe}_{2}$ PNS after $25 \mathrm{~h}$ stability test were summarized in Table 1 . The $\mathrm{Se} / \mathrm{Nb}$ ratio gradually decreases, and the content of $\mathrm{O}$ increases during the long-term electrochemical test, which illustrates that the $\mathrm{NbSe}_{2}$ is oxidized into niobium oxide during the electrochemical test. The $\mathrm{NbSe}_{2} \mathrm{NSs}$ exhibits $\mathrm{Nb}^{4+}$ in $\mathrm{NbSe}_{2}$ (Figure S3), and no oxygen was detected. Also, the introduction of $\mathrm{H}_{2} \mathrm{O}_{2}$ brought substantial oxygen group on the surface of $\mathrm{NbSe}_{2}$, causing a high $\mathrm{O} / \mathrm{Nb}$ ratio of 3.2 in the $\mathrm{NbSe}_{2}$ PNS. The $\mathrm{O} / \mathrm{Nb}$ ratio was further increased to 5.5 after electrochemical test due to the oxidization process of $\mathrm{NbSe}_{2}$ to $\mathrm{Nb}_{2} \mathrm{O}_{5}$ at acidic media with constant applied potential.

Table 1. The related XPS analysis results after standardization.

\begin{tabular}{ccccc}
\hline & $\mathbf{N b S e}_{2} \mathbf{~ N S s}$ & $\mathbf{N b S e}_{2}$ PNS & $\mathbf{N b S e}_{2}$ PNS after 50 cycles & $\mathbf{N b S e}_{\mathbf{2}}$ PNS after 25 h \\
\hline $\mathrm{Nb} 3 \mathrm{~d}$ & 0.285 & 0.149 & 0.178 & 0.126 \\
$\mathrm{Se} 3 \mathrm{~d}$ & 0.644 & 0.374 & 0.339 & 0.174 \\
$\mathrm{O} \mathrm{1s}$ & 0.071 & 0.476 & 0.482 & 0.696 \\
\hline
\end{tabular}


The electrocatalytic HER activities of the $\mathrm{NbSe}_{2}$ PNS/CF were investigated by linear-sweep voltammetry (LSV) using a standard three-electrode setup in $0.5 \mathrm{M} \mathrm{H}_{2} \mathrm{SO}_{4}$ solution with a scan rate of $5 \mathrm{mV} \mathrm{s}^{-1}$. For comparison, the reference commercial Pt-C (10 wt. \% Pt) was studied under the same condition. Figure 7a shows the LSV curves of various samples after IR compensation. Pure CF shows limited HER activity within the potential range of $-0.4 \sim 0.2 \mathrm{~V}$ (versus $\mathrm{RHE}$ ), whereas the Pt-C has the best catalytic performance. To achieve current densities of $-50 \mathrm{~mA} \mathrm{~cm}^{-2}$, the exfoliated porous $\mathrm{NbSe}_{2} \mathrm{PNS} / \mathrm{CF}$ requires an overpotential of $148 \mathrm{mV}$. In contrast, the $\mathrm{NbSe}_{2} \mathrm{NSs} / \mathrm{CF}$ without porous structure and the $\mathrm{NbSe}_{2}$ bulk exhibits an inferior HER activity with a larger overpotential of $242 \mathrm{mV}$ and $400 \mathrm{mV}$ to drive the hydrogen-evolution current of $-50 \mathrm{~mA} \mathrm{~cm}^{-2}$, respectively. Compared with the HER activities of the $\mathrm{NbSe}_{2} \mathrm{PNS} / \mathrm{CF}, \mathrm{NbSe}_{2} \mathrm{NSs} / \mathrm{CF}$ and $\mathrm{NbSe}_{2}$ bulk/CF, it can be concluded that the porous nanosheets structure of the single-layer $\mathrm{NbSe}_{2}$ PNS indeed improve the catalyzed HER activity. A similar phenomenon has been reported in ultra-thin and porous $\mathrm{MoSe}_{2}$ nanosheets [39].
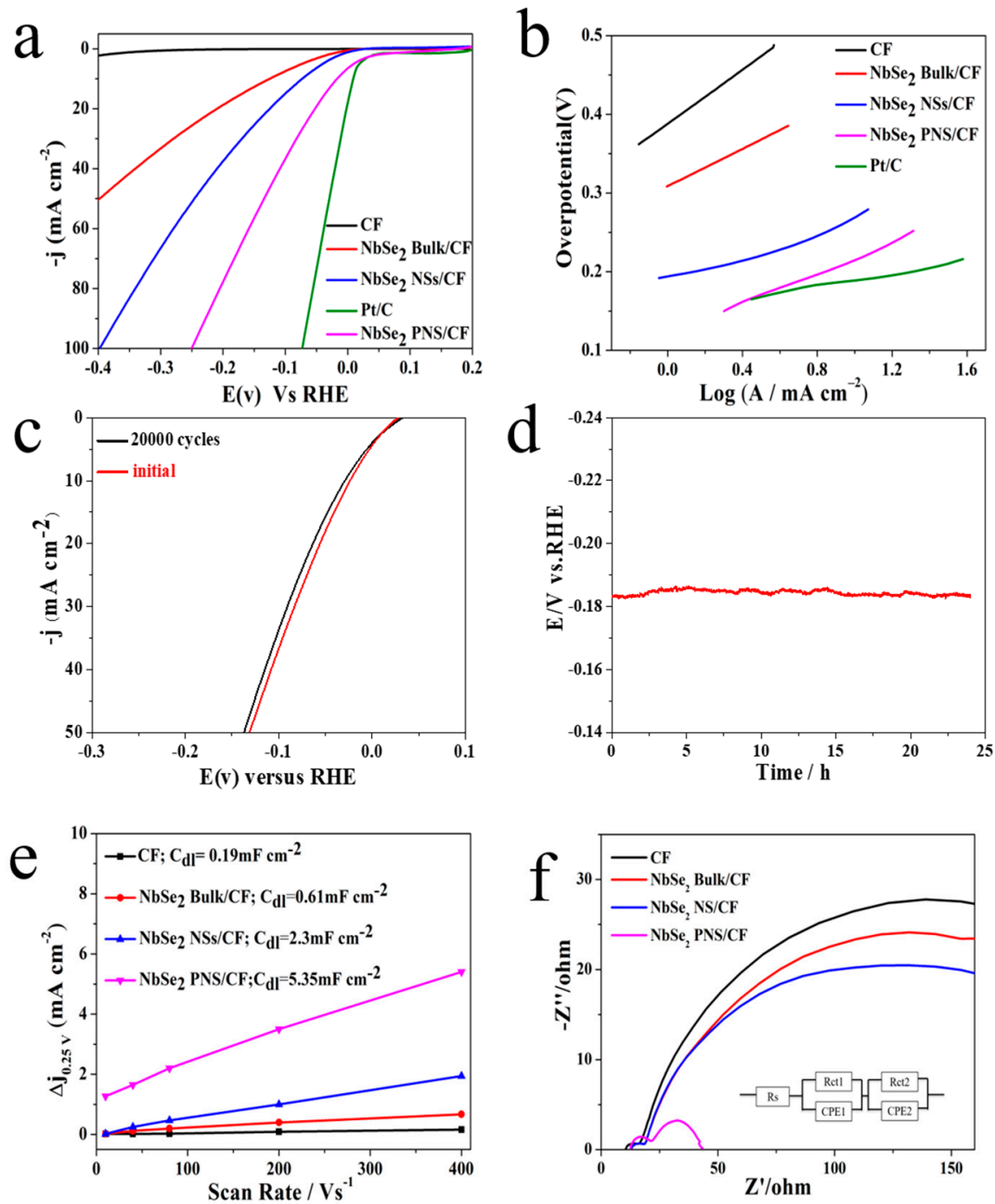

Figure 7. (a) LSV curves of the $\mathrm{NbSe}_{2} \mathrm{PNS} / \mathrm{CF}, \mathrm{NbSe}_{2} \mathrm{NSs} / \mathrm{CF}, \mathrm{NbSe}_{2}$ bulk/CF, $\mathrm{Pt}-\mathrm{C}$ and bare $\mathrm{CF}$ at a scan rate of $5 \mathrm{mV} \mathrm{s}^{-1}$. (b) Tafel plots of the $\mathrm{NbSe}_{2} \mathrm{PNS} / \mathrm{CF}, \mathrm{NbSe}_{2} \mathrm{NSs} / \mathrm{CF}, \mathrm{NbSe}_{2}$ bulk/CF, Pt-C and bare CF. (c) Polarization curve comparison between initial and after 20,000 cycles of the $\mathrm{NbSe}_{2}$ $\mathrm{PNS} / \mathrm{CF}$ at a scan rate of $50 \mathrm{mV} \mathrm{s}^{-1}$. (d) Chronopotentiometric curve recorded for the $\mathrm{NbSe}_{2} \mathrm{PNS} / \mathrm{CF}$ at a constant cathodic current density of $50 \mathrm{~mA} \mathrm{~cm}^{-2}$. (e) The slope of current density at open circuit potential (OCP) vs. scan rate. (f) Nyquist plots of $\mathrm{NbSe}_{2} \mathrm{PNS} / \mathrm{CF}, \mathrm{NbSe}_{2} \mathrm{NSs} / \mathrm{CF}, \mathrm{NbSe}_{2}$ bulk/CF and CF. 
To understand the high HER activity of the $\mathrm{NbSe}_{2} \mathrm{PNS} / \mathrm{CF}$, Tafel plots of various electrodes were studied (Figure $7 \mathrm{~b}$ ). The Tafel plots were derived from the quasi-static polarization curve to reflect the inherent mechanism of the HER process and the rate-determining step for the entire HER process. A smaller Tafel slope is referred to as a faster increase of hydrogen-generation rate [40]. The pure CF shows a large Tafel slope of $\sim 300 \mathrm{mV} \mathrm{dec}^{-1}$ in the $\eta$ range of $360-480 \mathrm{mV}$, which indicates that it is a less active HER catalyst. The Pt-C is the most active material with the smallest Tafel slope of $41 \mathrm{mV} \mathrm{dec}^{-1}$. The $\mathrm{NbSe}_{2} \mathrm{PNS} / \mathrm{CF}$ possesses a Tafel slope of $75.8 \mathrm{mV} \mathrm{dec}^{-1}$, which is smaller than those of 97.3 and $155 \mathrm{mV} \mathrm{dec}^{-1}$ for the $\mathrm{NbSe}_{2} \mathrm{NS} / \mathrm{CF}$ and $\mathrm{NbSe}_{2}$ bulk/CF, respectively, which demonstrates the more rapid HER kinetics of $\mathrm{NbSe}_{2} \mathrm{PNS} / \mathrm{CF}$. The Tafel slope of $\mathrm{NbSe}_{2} \mathrm{PNS} / \mathrm{CF}$ is either close to or even better than the records of the three-dimensional TMD-based electrocatalysts (Table S1), such as $\mathrm{MoS}_{2}-\mathrm{NbSe}_{2}$ hybrid nanobelts (101.2 $\mathrm{mV} \mathrm{dec}^{-1}$ ) [20], three-dimensional molybdenum sulfide sponges $\left(185 \mathrm{mV} \mathrm{dec}^{-1}\right)$ [29] and three-dimensional $\mathrm{MoS}_{2} / \mathrm{GO}$ frameworks $\left(86.3 \mathrm{mV} \mathrm{dec}^{-1}\right)$ [30]. The porous structure of the single-layer $\mathrm{NbSe}_{2}$ PNS can improve the catalytic activity toward better HER due to the additional edge sites along the margins of the hole. Furthermore, the unsaturated Se along the holes provides possible active sites for hydrogen-ion adsorption [28].

The excellent stability of electrocatalysts towards the HER is vital for future water-splitting systems. Figure 7c shows the continuous cycling performance of the $\mathrm{NbSe}_{2} \mathrm{PNS} / \mathrm{CF}$ electrode for 20,000 cycles at a scan rate of $50 \mathrm{mV} \mathrm{s}^{-1}$. At a current density of $-50 \mathrm{~mA} \mathrm{~cm}^{-2}$, the overpotential of the $\mathrm{NbSe}_{2} \mathrm{PNS} / \mathrm{CF}$ shows a slight increase after 20,000 cycles. Consequently, the $\mathrm{NbSe}_{2} \mathrm{PNS} / \mathrm{CF}$ exhibits an ultra-high activity and a satisfied long-term cycle stability. Figure $7 \mathrm{~d}$ shows the chronopotentiometric plot recorded for the $\mathrm{NbSe}_{2} \mathrm{PNS} / \mathrm{CF}$ at a constant current density of $-50 \mathrm{~mA} \mathrm{~cm}{ }^{-2}$. The potential of $\mathrm{NbSe}_{2}$ PNS/CF was maintained constant with little oscillation over $24 \mathrm{~h}$, suggesting the high durability of the $\mathrm{NbSe}_{2} \mathrm{PNS} / \mathrm{CF}$. The SEM and TEM images (Figure S5) show that the $\mathrm{NbSe}_{2} \mathrm{PNS}_{\text {maintain a } 2 \mathrm{D}}$ lamella structure and regular lattice fringes of $0.31 \mathrm{~nm}$. The $\mathrm{NbSe}_{2} \mathrm{PNS}$ after 20,000 cycles were surface partly oxidized to niobium pentoxide (Figure S4). The diffraction peaks were labelled as well numbers were assigned to the crystal planes of $\mathrm{Nb}_{2} \mathrm{O}_{5}$ (Figure S9, JCPDF 72-1121) while the diffraction peaks of $\mathrm{NbSe}_{2} \mathrm{PNS}$ were maintained the same $\left(15.0^{\circ}, 22.6^{\circ}\right.$ and $\left.29.2^{\circ}\right)$. However, the $\mathrm{NbSe}_{2} \mathrm{PNS} / \mathrm{CF}$ after 20,000 cycles still exhibited an ultra-high electrochemical activity, which illustrates that the $\mathrm{NbSe}_{2}$ PNS/CF electrodes have a great long-term stability. To further confirm the active catalytic species in the $\mathrm{NbSe}_{2} \mathrm{PNS} / \mathrm{CF}$ electrode, the LSV curves of $\mathrm{Nb}_{2} \mathrm{O}_{5}, \mathrm{NbSe}_{2} \mathrm{PNS} / \mathrm{CF}, \mathrm{NbSe}_{2} \mathrm{PNS} / \mathrm{CF}$ after 50 consecutive cyclic voltammetry sweeps and $\mathrm{NbSe}_{2} \mathrm{PNS} / \mathrm{CF}$ after $25 \mathrm{~h}$ stability test with the scan rate of $100 \mathrm{mV} / \mathrm{s}$ in $0.5 \mathrm{M} \mathrm{H}_{2} \mathrm{SO}_{4}$ were tested (see Figure S10). The XRD patterns of $\mathrm{NbSe}_{2} \mathrm{PNS} / \mathrm{CF}$, $\mathrm{NbSe}_{2} \mathrm{PNS} / \mathrm{CF}$ after 50 consecutive cyclic voltammetry sweeps and $\mathrm{NbSe}_{2}$ PNS/CF after $25 \mathrm{~h}$ stability test were shown in Figure S4. Combining the XPS (Table 1) and XRD results (Figure S4), it can be concluded that the $\mathrm{NbSe}_{2}$ PNS in the electrode surface was gradually oxidized to $\mathrm{Nb}_{2} \mathrm{O}_{5}$ during the whole electrochemical test. The pure $\mathrm{Nb}_{2} \mathrm{O}_{5}$ exhibits a weak HER performance, which illustrates that the $\mathrm{Nb}_{2} \mathrm{O}_{5}$ is not an active catalytic species for hydrogen evolution reaction. The LSV curve of $\mathrm{NbSe}_{2}$ $\mathrm{PNS} / \mathrm{CF}$ after 50 cycles almost coincided with the initial $\mathrm{NbSe}_{2} \mathrm{PNS} / \mathrm{CF}$, while the $\mathrm{NbSe}_{2} \mathrm{PNS} / \mathrm{CF}$ after long-term stability test showed little current attenuation compared with the initial $\mathrm{NbSe}_{2} \mathrm{PNS} / \mathrm{CF}$. The $\mathrm{NbSe}_{2} \mathrm{PNS} / \mathrm{CF}$ electrode exhibited the satisfied stability even when the electrode surface was gradually transferred to niobium oxide. Considering the low electrocatalytic performance of $\mathrm{Nb}_{2} \mathrm{O}_{5}$, the invariant electrocatalytic performance of $\mathrm{NbSe}_{2} \mathrm{PNS} / \mathrm{CF}$ electrode may be due to the high active of exposed $\mathrm{NbSe}_{2}$ catalyst.

Catalysis process is related to the interactions between the catalyst surface and the adsorbed species (reaction intermediates) [41]. Electrochemical active surface is also an important factor to reflect the electrocatalytic performance. The electrochemical double-layer capacitance $\left(C_{d 1}\right)$ is used to estimate the electrochemical active surface area for each system [42]. To measure the electrochemical capacitance of $\mathrm{CF}, \mathrm{NbSe}_{2}$ bulk/CF, $\mathrm{NbSe}_{2} \mathrm{NSs} / \mathrm{CF}$ and $\mathrm{NbSe}_{2} \mathrm{PNS} / \mathrm{CF}, \mathrm{CVs}$ with a potential range of $\pm 100 \mathrm{mV}$ versus open circuit potential (OCP), were scanned at 10, 40, 80, 200 and $400 \mathrm{mV} \mathrm{s}^{-1}$ (Figure S6). The OCP for $\mathrm{CF}, \mathrm{NbSe}_{2} \mathrm{NSs} / \mathrm{CF}$ and $\mathrm{NbSe}_{2} \mathrm{PNS} / \mathrm{CF}$ are $0.15 \mathrm{~V}, 0.21 \mathrm{~V}, 0.18 \mathrm{~V}$, respectively. Figure 7e shows the 
slope of the current density versus the scan rate. The measured $\mathrm{C}_{\mathrm{dl}}$ were plotted as a function of scan rate via a linear fitting. The $\mathrm{C}_{\mathrm{dl}}$ of $\mathrm{NbSe}_{2} \mathrm{PNS} / \mathrm{CF}$ is more than twice that of $\mathrm{NbSe}_{2} \mathrm{NSs} / \mathrm{CF}$ (5.35 versus $2.3 \mathrm{mF} \mathrm{cm}^{-2}$ ), whereas the $\mathrm{C}_{\mathrm{dl}}$ of the $\mathrm{NbSe}_{2}$ bulk/CF and pure $\mathrm{CF}$ is only 0.61 and $0.19 \mathrm{mF} \mathrm{cm}^{-2}$, respectively. These results show that $\mathrm{NbSe}_{2} \mathrm{PNS} / \mathrm{CF}$ possesses more HER active sites than that of the $\mathrm{NbSe}_{2} \mathrm{NSs} / \mathrm{CF}$ because more basal planes were exposed in this typical porous structure. Thus, this beneficial distinct feature leads to a higher HER activity. Electrochemical impedance spectroscopy (EIS) analysis was carried out to investigate the charge-transfer resistance $\left(\mathrm{R}_{\mathrm{ct}}\right)$ of different samples. Figure $7 \mathrm{f}$ shows Nyquist plots of $\mathrm{NbSe}_{2} \mathrm{PNS}, \mathrm{NbSe}_{2} \mathrm{NSs}$ and CF. The EIS profile can be fitted to two semicircles. The first high-frequency arches are related to the solid-solid interface resistance $\left(R_{c t 1}\right)$, the second semicircles in the lower frequency range are associated with the electron transfer at the solid/electrolyte interface $\left(\mathrm{R}_{\mathrm{ct} 2}\right)$ (inset in Figure $7 \mathrm{f}$ ) [43]. The $\mathrm{R}_{\mathrm{S}}$ values of $\mathrm{NbSe}_{2} \mathrm{PNS} / \mathrm{CF}, \mathrm{NbSe}{ }_{2} \mathrm{NSs} / \mathrm{CF}$, $\mathrm{NbSe}_{2}$ Bulk/CF and CF are similar (12 13 $\Omega$ ), and the $\mathrm{R}_{\mathrm{ct} 1}$ value in each electrode is not significantly different $\left(\mathrm{R}_{\mathrm{ct} 1}\right.$ is $7 \Omega$ for $\mathrm{NbSe}_{2} \mathrm{NSs} / \mathrm{CF}, \mathrm{NbSe}{ }_{2}$ Bulk/CF and CF; $\mathrm{R}_{\mathrm{ct} 1}$ is $10 \Omega$ for $\left.\mathrm{NbSe}_{2} \mathrm{PNS} / \mathrm{CF}\right) . \mathrm{NbSe}_{2}$ PNS possesses a small $R_{c t 2}$ of $6.3 \Omega$, which is significantly lower than that of $\mathrm{NbSe}_{2} \mathrm{NSs}$ of $191.7 \Omega$, $\mathrm{NbSe}_{2}$ bulk of $253.7 \Omega$, and CF of $298.4 \Omega$. The lower $\mathrm{R}_{\mathrm{ct}}$ indicates the rapid HER reaction kinetics, which may be attributed to the great conductivity and abundant active edge sites of the $\mathrm{NbSe}_{2} \mathrm{PNS}_{\text {. }}$

\section{Discussion and Conclusions}

Single-layer porous $\mathrm{NbSe}_{2}$ nanosheets have been prepared via double-sonication liquid-phase exfoliation with the assistance of $\mathrm{H}_{2} \mathrm{O}_{2}$. The single-layer porous $\mathrm{NbSe}_{2}$ nanosheets were loaded on the CF surface as efficient electrocatalytic electrodes for HER. Compared with the $\mathrm{NbSe}_{2} \mathrm{NSs} / \mathrm{CF}$ and pure carbon foam, the $\mathrm{NbSe}_{2}$ PNS/CF exhibited excellent HER catalytic properties in acidic electrolyte with a low overpotential ( $-50 \mathrm{~mA} \mathrm{~cm}^{-2}$ at an overpotential of $\sim 148 \mathrm{mV}$ ), and a small Tafel slope of $75.8 \mathrm{mV} \mathrm{dec}^{-1}$. The $\mathrm{NbSe}_{2}$ PNS/CF shows little deactivation in continuous CV testing up to 20,000 cycles. These results suggest the promise of this novel $\mathrm{NbSe}_{2}$ PNS/CF electrode in electrochemical water splitting for hydrogen production. The enhanced HER performance is attributed to the accelerated electrochemical reaction that results from the increased edge active sites. The good HER performance of the $\mathrm{NbSe}_{2} \mathrm{PNS} / \mathrm{CF}$ is attributed to the increased conductivity and the faster electron-transfer rate. This work provides a new insight into the future construction of high-performance HER electrocatalysts.

Supplementary Materials: The following are available online at http://www.mdpi.com/2079-4991/9/5/751/s1, Figure S1: SEM images of the carbon foam, $\mathrm{NbSe}_{2}$ pristine powder, $\mathrm{NbSe}_{2} \mathrm{NSs}$ and $\mathrm{NbSe}_{2}$ PNS.; Figure S2: Typical AFM image and corresponding thickness analysis of $\mathrm{NbSe}_{2} \mathrm{NSs}$, TEM and HRTEM images of $\mathrm{NbSe}_{2} \mathrm{NSs}_{\text {; }}$ Figure S3: The high-resolution XPS spectra of $\mathrm{NbSe}_{2} \mathrm{NSs} S \mathrm{Se} 3 \mathrm{~d}$ and $\mathrm{Nb} 3 \mathrm{~d}$; Figure S4: XRD patterns of $\mathrm{NbSe}_{2} \mathrm{PNS}_{\text {, }}$ NbSe2 PNS after 50 consecutive cycle voltammetry sweeps and $\mathrm{NbSe}_{2}$ after $25 \mathrm{~h}$ stability test PNS; Figure S5: The SEM image of $\mathrm{NbSe}_{2}$ PNS after stability test and the TEM image of $\mathrm{NbSe}_{2}$ PNS after stability test; Figure S6: Double-layer capacitance measurements for determining the electrochemically active surface areas of the $C F$, $\mathrm{NbSe}_{2} \mathrm{NSs} / \mathrm{CF}$ and $\mathrm{NbSe}_{2}$ PNS/CF; Figure S7: High-resolution XPS spectrum of O 1s of $\mathrm{NbSe}_{2}$ PNS; Figure S8: High-resolution XPS spectrum of $\mathrm{NbSe}_{2}$ PNS after 50 consecutive cyclic voltammetry sweeps; Figure S9: XRD patterns of $\mathrm{Nb}_{2} \mathrm{O}_{5}$; Figure S10: LSV curves of $\mathrm{Nb}_{2} \mathrm{O}_{5}, \mathrm{NbSe}_{2}$ PNS/CF, NbSe 2 PNS/CF after 50 consecutive cyclic voltammetry sweeps and $\mathrm{NbSe}_{2}$ PNS/CF after $25 \mathrm{~h}$ stability test. Table S1: Comparison of HER performance in acid medium for $\mathrm{NbSe}_{2} \mathrm{PNS} / \mathrm{CF}$ with other recently reported non-noble-metal related HER catalysts.

Author Contributions: Conceptualization, J.W.; Funding acquisition, G.Y.; Methodology, X.L.; Supervision, G.Y.; Writing—original draft, J.W.; Writing—review \& editing, Y.L.

Funding: This research was funded by the Program of National Basic Research Program of China (Project No. 2014CB931700) and the Science and Technology Planning Project of Guangdong Province (Project No. 2017B090918002) and State Key Laboratory of Optoelectronic Materials and Technologies.

Acknowledgments: The authors gratefully thank the State Key Laboratory of Optoelectronic Materials and Technologies for the instrument support.

Conflicts of Interest: There are no conflict to declare. 


\section{References}

1. Cortright, R.D.; Davda, R.R.; Dumesic, J.A. Hydrogen from Catalytic Reforming of Biomass-derived Hydrocarbons in Liquid Water. Nature 2002, 33, 964-967. [CrossRef]

2. Wang, X.; Kolen'ko, Y.; Bao, X.; Kovnir, K.; Liu, L. One-Step Synthesis of Self-Supported Nickel Phosphide Nanosheet Array Cathodes for Efficient Electrocatalytic Hydrogen Generation. Angew. Chem. Int. Ed. 2015, 54, 8188-8192. [CrossRef] [PubMed]

3. Jiao, Y.; Zheng, Y.; Jaroniec, M.; Qiao, S.Z. Design of Electrocatalysts for Oxygen-and Hydrogen-involving Energy Conversion Reactions. Chem. Soc. Rev. 2015, 44, 2060-2086. [CrossRef]

4. Wang, X.; Li, W.; Xiong, D.; Petrovykh, D.; Liu, L. Bifunctional Nickel Phosphide Nanocatalysts Supported on Carbon Fiber Paper for Highly Efficient and Stable Overall Water Splitting. Adv. Fun. Mater. 2016, 26, 4067-4077. [CrossRef]

5. Zheng, Y.; Jiao, Y.; Jaroniec, M.; Qiao, S.Z. Advancing the Electrochemistry of the Hydrogen-Evolution Reaction Through Combining Experiment and Theory. Angew. Chem. Int. Ed. 2015, 54, 52-65. [CrossRef]

6. Subbaraman, R.; Tripkovic, D.; Chang, K.C.; Strmcnik, D.; Paulikas, A.P.; Hirunsit, P.; Chan, M.; Greeley, J.; Stamenkovic, V.; Markovic, N.M. Trends in Activity for the Water Electrolyser Reactions on 3d M (Ni,Co,Fe,Mn) Hydr(oxy)oxide Catalysts. Nat. Mater. 2012, 11, 550-557. [CrossRef]

7. Gao, M.R.; Liang, J.X.; Zheng, Y.R.; Xu, Y.F.; Jiang, J.; Gao, Q.; Li, J.; Yu, S.H. An Efficient Molybdenum Disulfide/Cobalt Diselenide Hybrid Catalyst for Electrochemical Hydrogen Generation. Nat. Commun. 2015, 6, 5982-5984. [CrossRef] [PubMed]

8. Tang, H.; Hessel, C.M.; Wang, J.; Yang, N.; Yu, R.; Zhao, H.; Wang, D. Two-Dimensional Carbon Leading to New Photoconversion Processes. Chem. Soc. Rev. 2014, 43, 4281-4299. [CrossRef]

9. Ma, Z.; Zhao, Q.; Li, J.; Tang, B.; Zhang, Z.; Wang, X. Three-Dimensional Well-Mixed/Highly-Densed NiS-CoS Nanorod Arrays: An Efficient and Stable Bifunctional Electrocatalyst for Hydrogen and Oxygen Evolution Reactions. Electrochim. Acta 2018, 260, 82-91. [CrossRef]

10. Seh, Z.W.; Kibsgaard, J.; Dickens, C.F.; Chorkendorff, I.; Nørskov, J.K.; Jaramillo, T.F. Combining Theory and Experiment in Electrocatalysis: Insights into Materials Design. Science 2017, 355, 146-148. [CrossRef] [PubMed]

11. Lin, Z.; Li, J.; Zheng, Z.; Li, L.; Yu, L.; Wang, C.; Yang, G. A Floating Sheet for Efficient Photocatalytic Water Splitting. Adv. Energy Mater. 2016, 6, 1600510-1600516. [CrossRef]

12. Bi, W.; Li, X.; Zhang, L.; Jin, T.; Zhang, L.; Zhang, Q.; Luo, Y.; Wu, C.; Xie, Y. Molecular Co-Catalyst Accelerating Hole Transfer for Enhanced Photocatalytic $\mathrm{H}_{2}$ Evolution. Nat. Commun. 2015, 6, 8647-8651. [CrossRef]

13. Ma, Z.; Meng, H.; Wang, M.; Tang, B.; Li, J.; Wang, X. Porous Ni-Mo-S Nanowire Network Film Electrode as High-Efficiency Bifunctional Electrocatalyst for Overall Water Splitting. ChemElectroChem 2017, 4, 1-9. [CrossRef]

14. Ma, Z.; Li, R.; Wang, M.; Meng, H.; Zhang, F.; Bao, X.; Tang, B.; Wang, X. Self-Supported Porous Ni-Fe-P Composite as An Efficient Electrocatalyst for Hydrogen Evolution Reaction in Both Acidic and Alkaline Medium. Electrochim. Acta 2016, 219, 194-203. [CrossRef]

15. Lukowski, M.A.; Daniel, A.S.; Meng, F.; Forticaux, A.; Li, L.; Jin, S. Enhanced Hydrogen Evolution Catalysis from Chemically Exfoliated Metallic $\mathrm{MoS}_{2}$ Nanosheets. J. Am. Chem. Soc. 2013, 135, 10274-10277. [CrossRef]

16. Ma, G.; He, Y.; Wang, M.; Zhu, F.; Tang, B.; Wang, X. An Efficient Route for Catalytic Activity Promotion via Hybrid Electrodepositional Modification on Commercial Nickel Foam for Hydrogen Evolution Reaction in Alkaline Water Electrolysis. Appl. Surf. Sci. 2014, 313, 512-523. [CrossRef]

17. Kibsgaard, J.; Chen, Z.; Reinecke, B.N.; Jaramillo, T.F. Engineering the Surface Structure of $\mathrm{MoS}_{2}$ to Preferentially Expose Active Edge Sites for Electrocatalysis. Nat. Mater. 2012, 11, 963-969. [CrossRef]

18. Voiry, D.; Yamaguchi, H.; Li, J.; Silva, R.; Alves, D.C.; Fujita, T.; Chen, M.; Asefa, T.; Shenoy, V.B.; Eda, G. Enhanced Catalytic Activity in Strained Chemically Exfoliated $\mathrm{WS}_{2}$ Nanosheets for Hydrogen Evolution. Nat. Mater. 2013, 12, 850-855. [CrossRef]

19. Voiry, D.; Fullon, R.; Yang, J.; De, C.C.; Kappera, R.; Bozkurt, I.; Kaplan, D.; Lagos, M.J.; Batson, P.E.; Gupta, G. The Role of Electronic Coupling Between Substrate and 2D MoS 2 Nanosheets in Electrocatalytic Production of Hydrogen. Nat. Mater. 2016, 15, 1003-1009. [CrossRef] 
20. Zhao, B.; Huang, J.; Fu, Q.; Yang, L.; Zhang, J.; Xiang, B. $\mathrm{MoS}_{2} / \mathrm{NbSe}_{2}$ Hybrid Nanobelts for Enhanced Hydrogen Evolution. J. Electrochem. Soc. 2016, 163, 384-387. [CrossRef]

21. Xi, X.; Zhao, L.; Wang, Z.; Berger, H.; Forró, L.; Shan, J.; Mak, K.F. Strongly Enhanced Charge-Density-Wave Order in Monolayer NbSe 2 . Nat. Nanotech. 2015, 10, 765-769. [CrossRef] [PubMed]

22. Chi, X.; Ambrosi, A.; Lazar, P.; Sofer, Z.; Pumera, M. Electrocatalysis of Layered Group 5 Metallic Transition Metal Dichalcogenides $\left(\mathrm{MX}_{2}, \mathrm{M}=\mathrm{V}, \mathrm{Nb}\right.$, and Ta; $\mathrm{X}=\mathrm{S}$, Se, and Te). J. Mater. Chem. A 2016, 4, 14241. [CrossRef]

23. Tang, H.; Cao, K.; Wu, Q.; Li, C.; Yang, X.; Yan, X. Synthesis and Tribological Properties of Copper Matrix Solid Self-Lubricant Composites Reinforced with $\mathrm{NbSe}_{2}$ Nanoparticles. Cryst. Res. Technol. 2011, 46, $195-200$. [CrossRef]

24. Sipos, B.; Kusmartseva, A.F.; Akrap, A.; Berger, H.; Forro, L.; Tutis, E. From Mott State to Superconductivity in $1 \mathrm{~T}-\mathrm{TaS}_{2}$. Nat. Mater. 2008, 7, 960-965. [CrossRef] [PubMed]

25. Calandra, M.; Mazin, I.I.; Mauri, F. Effect of Dimensionality on the Charge-Density-Wave in Few-Layers 2H-NbSe 2 . Phys. Rev. B 2009, 80, 241108. [CrossRef]

26. Park, S.K.; Chung, D.Y.; Ko, D.; Sung, Y.; Piao, Y. Three-Dimensional Carbon Foam/N-Doped Graphene@MoS Hybrid Nanostructures as Effective Electrocatalysts for the Hydrogen Evolution Reaction. J. Mater. Chem. A 2016, 4, 12720-12725. [CrossRef]

27. Tang, H.; Dou, K.; Kaun, C.C.; Kuang, Q.; Yang, S. MoSe 2 Nanosheets and Their Graphene Hybrids: Synthesis, Characterization and Hydrogen Evolution Reaction Studies. J. Mater. Chem. A 2013, 2, 360-364. [CrossRef]

28. Wang, H.; Lu, Z.; Xu, S.; Kong, D.; Cha, J.J.; Zheng, G.; Hsu, P.C.; Yan, K.; Bradshaw, D.; Prinz, F.B. Electrochemical Tuning of Vertically Aligned $\mathrm{MoS}_{2}$ Nanofilms and Its Application in Improving Hydrogen Evolution Reaction. Proc. Nat. Acad. Sci. USA 2013, 110, 19701-19706. [CrossRef]

29. Chang, Y.; Wu, F.; Chen, T.; Hsu, C.; Chen, C.; Wiryo, F.; Wei, K.; Chiang, C.; Li, L. Three-Dimensional Molybdenum Sulfide Sponges for Electrocatalytic Water Splitting. Small 2014, 10, 895-900. [CrossRef] [PubMed]

30. Zhou, W.; Zhou, K.; Hou, D.; Liu, X.; Li, G.; Sang, Y.; Liu, H.; Li, L.; Chen, S. Three-Dimensional Hierarchical Frameworks Based on $\mathrm{MoS}_{2}$ Nanosheets Self-Assembled on Graphene Oxide for Efficient Electrocatalytic Hydrogen Evolution. ACS Appl. Mater. Interf. 2014, 6, 21534-21540. [CrossRef] [PubMed]

31. Santos, H.M.; Lodeiro, C.; Capelo-Martínez, J.L. The Power of Ultrasound. In Ultrasound in Chemistry: Analytical Applications; Capelo-Martínez, J.L., Ed.; Wiley: Weinheim, Germany, 2009; Volume 1, pp. 15-16.

32. Backes, C.; Szydlowska, B.M.; Harvey, A.; Yuan, S.; Vega-Mayoral, V.; Davies, B.R.; Zhao, P.L.; Hanlon, D.; Santos, E.; Katsnelson, M.I. Production of Highly Monolayer Enriched Dispersions of Liquid-Exfoliated Nanosheets by Liquid Cascade Centrifugation. ACS Nano 2016, 10, 1589-1601. [CrossRef]

33. Khan, U.; Neill, A.O.; Lotya, M.; De, S.; Coleman, J.N. High-Concentration Solvent Exfoliation of Graphene. Small 2010, 6, 864-871. [CrossRef]

34. Lotya, M.; King, P.J.; Khan, U.; De, S.; Coleman, J.N. High-Concentration, Surfactant-Stabilized Graphene Dispersions. ACS Nano 2010, 4, 3155-3162. [CrossRef]

35. Smith, R.J.; King, P.J.; Lotya, M.; Wirtz, C.; Khan, U.; De, S.; Neill, A.O.; Duesberg, G.S.; Grunlan, J.C.; Moriarty, G. Large-Scale Exfoliation of Inorganic Layered Compounds in Aqueous Surfactant Solutions. Adv. Mater. 2011, 23, 3944-3948. [CrossRef]

36. Chung, D.Y.; Yoo, J.M.; Park, S.; Jung, G.Y.; Kang, J.S.; Ahn, C.Y.; Kwak, S.K.; Sung, Y. Edge-Terminated MoS Nanoassembled Electrocatalyst via In Situ Hybridization with 3D Carbon Network. Small 2018, 14, 1802191. [CrossRef] [PubMed]

37. Yu, Y.; Nam, G.; He, Q.; Wu, X.; Liu, Z.; Gu, L.; Du, Y.; Huang, W.; Zhang, H. High Phase-Purity $1 \mathrm{~T}^{\prime}-\mathrm{MoS}_{2}-$ and $1 \mathrm{~T}^{\prime}-\mathrm{MoSe}_{2}$-Layered Crystals. Nat. Chem. 2018, 10, 638-643. [CrossRef]

38. Jin, Y.O.; Ji, H.L.; Han, S.W.; Chae, S.S.; Bae, E.J.; Kang, Y.H.; Choi, W.J.; Song, Y.C.; Lee, J.O.; Hong, K.B. Chemically Exfoliated Transition Metal Dichalcogenide Nanosheet-based Wearable Thermoelectric Generators. Energy Environ. Sci. 2016, 9, 1696-1705.

39. Lei, Z.; Xu, S.; Wu, P. Ultra-Thin and Porous $\mathrm{MoSe}_{2}$ Nanosheets: Facile Preparation and Enhanced Electrocatalytic Activity Towards Hydrogen Evolution Reaction. Phys. Chem. Chem. Phys. 2016, 18, 70-74. [CrossRef] [PubMed]

40. Li, Y.; Wang, H.; Xie, L.; Liang, Y.; Hong, G.; Dai, H. MoS 2 Nanoparticles Grown on Graphene: An Advanced Catalyst for the Hydrogen Evolution Reaction. J. Am. Chem. Soc. 2011, 133, 7296-7299. [CrossRef] 
41. Song, F.; Hu, X. Ultrathin Cobalt-Manganese Layered Double Hydroxide is An Efficient Oxygen Evolution Catalyst. J. Am. Chem. Soc. 2014, 136, 16481-16484. [CrossRef]

42. Gao, Y.Q.; Liu, X.Y.; Yang, G.W. Amorphous Mixed-Metal Hydroxide Nanostructures for Advanced Water Oxidation Catalysts. Nanoscale 2016, 8, 5015-5023. [CrossRef]

43. Mccrory, C.C.; Jung, S.; Peters, J.C.; Jaramillo, T.F. Benchmarking Heterogeneous Electrocatalysts for the Oxygen Evolution Reaction. J. Am. Chem. Soc. 2013, 135, 16977-16987. [CrossRef]

(C) 2019 by the authors. Licensee MDPI, Basel, Switzerland. This article is an open access article distributed under the terms and conditions of the Creative Commons Attribution (CC BY) license (http://creativecommons.org/licenses/by/4.0/). 